\title{
Numerical investigation of wavelet features in rotating detonations with a two-step induction- reaction model
}

\author{
Honghui Teng ${ }^{a}$, Lin Zhou ${ }^{b}$, Pengfei Yang ${ }^{c, d, *}$, Zonglin Jiang ${ }^{c, d}$ \\ a Department of Mechanics, School of Aerospace Engineering, Beijing Institute of Technology, Beijing, 100081, China \\ b State Key Laboratory of Laser Propulsion and Application, Beijing Power Machinery Research Institute, Beijing, \\ 100074, China \\ c State Key Laboratory of High Temperature Gas Dynamics, Institute of Mechanics, Chinese Academy of Sciences, \\ Beijing, 100190, China \\ d School of Engineering Sciences, University of Chinese Academy of Sciences, Beijing, 100049, China
}

\section{H I G H L I G H T S}

- An increase in the stagnation temperature generates more rotating detonations.

- The variation of heat release rate also changes the wavelet number.

- The height and pressure of the detonation front oscillates periodically.

- The wavelet features in rotating detonation is sensitive to the ignition patterns.

\section{A R T I C L E I N F O}

\section{Article history:}

Received 22 March 2019

Received in revised form

8 December 2019

Accepted 11 December 2019

Available online $\mathrm{xxx}$

\section{Keywords:}

Rotating detonation

Induction-reaction kinetics

Wavelet pattern

Oscillations

\begin{abstract}
A B S T R A C T
The wavelet features of rotating detonation waves (RDWs) are numerically investigated using Euler equations and a two-step induction-reaction model. The effects of the inflow stagnation temperature $T_{s t}$ and the heat release rate $k_{R}$ on the number, height and intensity of the RDWs are discussed in this study. An increase in the stagnation temperature results in more detonation waves in a combustion chamber, which indicates the number of RDWs is sensitivity to the thermodynamic state of the reactants. As the heat release rate decreases, the number of detonation wave decreases and an unstable wavelet pattern is observed. This is represented as the oscillation in height and intensity of the detonation. In addition, some numerical cases are performed to determine the effects of ignition patterns on the number of RDWs. The features of the flow fields are analyzed using varied inflow stagnation temperature and initiation patterns, identifying the co-existence of different wavelet configurations.
\end{abstract}

(C) 2019 Hydrogen Energy Publications LLC. Published by Elsevier Ltd. All rights reserved.

\footnotetext{
* Corresponding author. State Key Laboratory of High Temperature Gas Dynamics, Institute of Mechanics, Chinese Academy of Sciences, Beijing, 100190, China.

E-mail address: young1505@foxmail.com (P. Yang). 


$\begin{array}{ll}\text { Nomenclature } \\ \text { RDW } & \text { Rotating detonation wave } \\ \mathrm{RDE} & \text { Rotating detonation engine } \\ \mathrm{C}-\mathrm{J} & \text { Chapman-Jouguet } \\ \xi & \text { reaction variable of the induction zone } \\ \lambda & \text { reaction variable of the reaction zone } \\ \gamma & \text { specific heat ratio } \\ \mathrm{Q} & \text { chemical energy release } \\ k_{\mathrm{I}} & \text { Pre-exponential factor of the induction zone } \\ E_{\mathrm{I}} & \text { Activation energy of the induction zone } \\ k_{\mathrm{R}} & \text { Pre-exponential factor of the reaction zone } \\ E_{\mathrm{R}} & \text { Activation energy of the reaction zone } \\ \mathrm{T}_{\mathrm{S}} & \text { Post-shock temperature of Chapman-Jouguet } \\ & \text { detonation } \\ \mathrm{T}_{\mathrm{st}} & \text { Stagnation temperature } \\ p_{\mathrm{st}} & \text { Stagnation pressure } \\ p_{\mathrm{w}} & \text { Pressure on the combustion chamber head } \\ p_{\mathrm{cr} 1} & \text { Critical pressure of subsonic exhaust } \\ p_{\mathrm{cr} 2} & \text { Critical pressure of supersonic exhaust } \\ A_{\mathrm{t}} & \text { Area of the micro-nozzle throat } \\ A_{\mathrm{w}} & \text { Area of the micro-nozzle exit }\end{array}$

Introduction

A supersonic detonation compresses the premixed reactants using a leading shock to achieve spontaneous ignition and self-sustained propagation. In recent years, detonation-based combustion has received increased interest because the thermodynamic efficiency is higher than traditional deflagration-based combustion system. Many types of heat engines such as the PDEs have been widely investigated to operate the detonation combustion in a combustor [1]. However, the PDE operating frequency is dependent on cycle timing and usually limited to a low value as the repeated ignition and fill. Hence, recent significant studies have almost concentrated on RDEs [2-7]. The premixed reactants are continually fed into an annular combustor allowing for a detonation to continually rotate circumferentially. For the continual operation, the operating frequency of RDEs is higher than the PDE and the performance of RDEs is more stable [4-6].

The premixed reactants are injected axially at the head end of the chamber, where one or more detonations propagate circumferentially in an annular combustor. The wave structures in RDEs consist of detonations, deflagrations and oblique shocks, which are controlled by the reactants thermodynamics parameters and the geometric scale of the combustor. The inner and outer radii are two key parameters determining the detonation wavelet features in an annular chamber. Schwer and Kailasanath [8] investigated the effect of the chamber's outer radius on the flow field of the RDW and found that the performance became more unsteady with an increase in the outer radius. That is to say, the chamber size has a relatively large influence on the flow field structures, especially for a large combustor width. Zhou and Wang [9] demonstrated that the variation in the flow field along the radial direction was negligible when the chamber width was small, but increasingly became larger as the chamber width increased using the numerical results. The computational and experimental results by Katta et al. [10] shown that the effect of the compression and expansion waves on the evolving of a rotating detonation varying the outer radii. In addition, the effects of the injection conditions on propagation operations and instabilities of RDEs were investigated experimentally. Various propagation modes of H2/Air RDEs were obtained by adjusting the mass flow rate [11]. More importantly, the number of RDWs could be changing even if maintained a constant mass flow rate. Concurrently, the similar phenomena were observed in the experimental results [12], which showed that an instantaneous shift from one operating mode to another, although the reason behind such a fact was still unknown. Ma et al. $[13,14]$ analyzed the initiation characteristics of rotating detonation wave using hydrogen/air mixture and found that the operating characteristic of the engine model was independent of the ignition device, but the injection area significantly affected the pressure and stability of the detonation wave. Considering the variable air-inlet slot and the equivalence ratio, the pressure and velocity characteristics of detonation wave were analyzed thoroughly $[15,16]$. Subsequently, many significant studies [17-21] have evaluated the effects of the injection conditions, ignitions methods and geometric configuration on complex propagation patterns.

The radial width is typically small compared to the azimuthal and axial dimension and the variation of the RDW flow field is generally little in the radial direction. In order to lessen the computation load, complexity and improve precision, a simplified model of two-dimensional (2D) study of the RDWs can be conducted numerically and theoretically by unwrapping the annular combustor. This approximation has been widely used in recent studies and has provided many important progress on the flow structures, stability and dynamics of RDWs. Hayashi et al. [22,23] numerically investigated the RDWs using a premixed hydrogen-oxygen mixture and analyzed the effect of the inlet pressure, Mach number, micro-nozzle area ratio and ignition energy on the rotation detonation limit and thrust performance. A detailed description on the characteristics of the flow field and detonation structure have been presented by Schwer et al. [24,25] and the effects of the injection pressure, engine size and inlet model on the performance are also investigated using a hydrogen/ hydrocarbon-air RDE. The instabilities of 2D RDWs have been analyzed and characterized by the generation of transverse waves and high-frequency oscillations of the detonation height/pressure [26,27].

Although the formation of multiple wavelets is frequently observed, there still exist several puzzling problems, for example, the wave number in detonation chamber cannot be predicted based on the inflow parameters. Previous studies $[2,3,28,29]$ have mainly focused on the inflow stagnation pressure and the mass flow rate, while the effects of temperature are still unclear. The generalizing dependences of the wave number on the inflow stagnation temperature $T_{\text {st }}$ are shown in this study. In addition, to demonstrate the co- 
existence of several different structures, the effects of the ignition patterns and the type of fuel by varying the heat release rate $k_{\mathrm{R}}$ are also analyzed.

\section{Numerical model and methods}

The annular combustor is unwrapped into two dimensions because the radial dimension is usually smaller than the azimuthal and axial dimensions. A schematic of the 2D initial flow field is shown in Fig. 1. The ignition zone is a ChapmanJouguet (C-J) detonation wave, which is first computed in a straight channel with the both opening ends.

Following previous studies [30-32], reactive Euler equations are used as the governing equations to simulate the detonation flow field. To implement the two-step model for chain-branching kinetics [33], two additional reaction variables are introduced: one is used for the induction, $\xi$, and the other for the heat release, $\lambda$. The transport equations of these reaction variables can be written as:

$\frac{\partial(\rho \xi)}{\partial t}+\frac{\partial(\rho u \xi)}{\partial x}+\frac{\partial(\rho v \xi)}{\partial y}=H(1-\xi) \rho k_{I} \exp \left[E_{I}\left(\frac{1}{T_{S}}-\frac{1}{T}\right)\right]$

$\frac{\partial(\rho \lambda)}{\partial t}+\frac{\partial(\rho u \lambda)}{\partial x}+\frac{\partial(\rho v \lambda)}{\partial y}=[1-H(1-\xi)] \rho(1-\lambda) k_{R} \exp \left[-\frac{E_{R}}{T}\right]$

The Heaviside step function is:

$H(1-\xi)= \begin{cases}1, & \text { if } \xi \leq 1 \\ 0, & \text { if } \xi>1\end{cases}$

The specific total energy is thus expressed as:

$e=\frac{p}{\rho(\gamma-1)}+\frac{1}{2}\left(u^{2}+v^{2}\right)-\lambda Q$

The variables $\rho, u, v, p, e$ and $Q$ are the density, $x$-direction velocity, $y$-direction velocity, pressure, specific total energy,

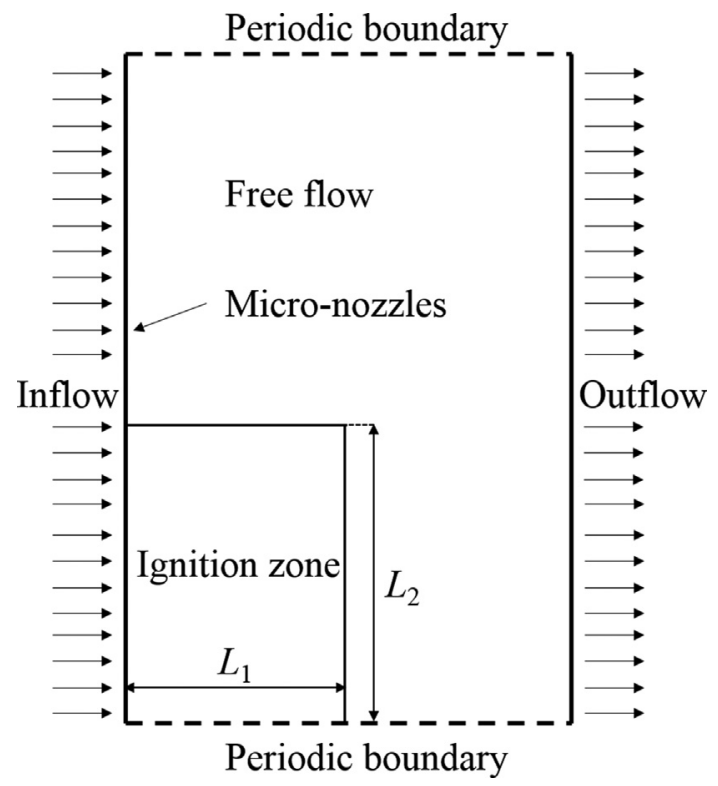

Fig. 1 - Schematic of the initiation of rotating detonation waves. and the amount of chemical heat release, respectively. All variables are dimensionless by referencing the uniform unburned state as follows:

$\rho=\frac{\tilde{\rho}}{\rho_{0}}, \quad p=\frac{\tilde{p}}{p_{0}}, \quad \mathrm{~T}=\frac{\tilde{\mathrm{T}}}{\mathrm{T}_{0}}, \quad \mathrm{u}=\frac{\tilde{u}}{\sqrt{\mathrm{RT} \mathrm{T}_{0}}}$

The dispersion-controlled dissipation scheme [34] with a 3rd Runge-Kutta algorithm are used to approximate the solution of the governing equations.

A premixed $\mathrm{H}_{2}$-air mixture is injected through the left boundary which is assumed to consist of many micro-nozzles. The injector area ratio $A_{w} / A_{t}$ is set to 2.0, where $A_{w}$ is the injection area of the combustor and $A_{t}$ is the area of the injector throats of the micro-nozzles. The injection parameters at the inlet are computed using the stagnation pressure/temperature $\left(p_{\mathrm{st}}\right.$ and $\mathrm{T}_{\mathrm{st}}$, ) and the pressure $p_{\mathrm{w}}$ near the inlet injection plane:

(1) If $p_{\mathrm{w}} \geq p_{\mathrm{st}}$, the combustible mixtures cannot be injected into the chamber and the boundary is treated as a wall.

(2) If $p_{\mathrm{cr} 1} \leq p_{\mathrm{w}} \leq p_{\mathrm{st}}$, the flow through injection wall is subsonic and the inflow parameters are obtained from the isentropic equations.

(3) If $p_{\mathrm{cr} 2} \leq p_{\mathrm{w}} \leq p_{\mathrm{cr} 1}$, the throat is choked and the mixture is injected at subsonic speed.

(4) If $p_{\mathrm{w}} \leq p_{\mathrm{cr} 2}$, the flow is not affected by the products pressure and the mixture is injected at supersonic speed.

The critical pressure is defined in terms of the pressure $p_{\text {st }}$ and the injected area ratio $A_{w} / A_{t}$ as:

$p_{\mathrm{cr} 1}=p_{\mathrm{st}}\left(1+\frac{\gamma-1}{2} M a_{2}^{2}\right)^{-\frac{\gamma}{\gamma-1}}$

$p_{\text {temp }}=p_{\text {st }}\left(1+\frac{\gamma-1}{2} M a_{1}^{2}\right)^{-\frac{\gamma}{\gamma-1}}$

$p_{\mathrm{cr} 2}=p_{\text {temp }}\left[1+\frac{2 \gamma}{\gamma+1}\left(\mathrm{Ma}_{1}^{2}-1\right)\right]$

The injection Mach number $\left(M a_{1} \geq 1, M a_{2} \leq 1\right)$ is defined as:

$\frac{A_{\mathrm{w}}}{A_{\mathrm{t}}}=\frac{1}{\mathrm{Ma}}\left[\frac{2+(\gamma-1) M a^{2}}{\gamma+1}\right]^{\frac{\gamma+1}{2(\gamma-1)}}$

The upper and lower boundary are interconnected using the periodic conditions. The outflow boundary condition is an extrapolated outflow [35] and the values $Y_{b}(\rho, u, v, p, \xi, \lambda)$ of the boundary are calculated as:

$\mathrm{Y}_{\mathrm{b}}=\mathrm{Y}_{1}(1-r)+\mathrm{Y}_{\mathrm{e}} r$

where $r$ is the relaxation rate coefficient, which is set to 0.05 , and $Y_{1}$ is the current value in the first cell near the boundary. The value $Y_{e}$ is the extrapolation limit and is equal to the ambient fluid parameters.

The dimensionless stagnation pressure $p_{\text {st }}$ is fixed at 20 and the dimensionless stagnation temperature $T_{\text {st }}$ varies to obtain different structural configurations of the RDWs. The default ignition region is set at $L_{1} \times L_{2}=192 \times 150$, and further studies 
on the initiation patterns are described in Possibility of multipattern induced by initiation.

\section{Numerical results and discussion}

\section{Modeling the chemical reactions using two-step kinetics}

Numerical simulations of detonation waves require solving the reactive Euler equations with an accurate chemical reaction model for the combustible mixtures. The ideal method entails solving the conservation equations and rate equations of all known species. However, the computational costs are very high, especially for the simulation of a detonation including the coupling of a strong shock and rapid chemical reaction. To present the physical properties of the detonation combustion quickly and accurately, a simplified chemical reaction model is required. A two-step reaction kinetic model consisting of a thermally neutral induction step followed by a main heat release reaction layer, can be considered. This section focus on the modeling and optimization of the twostep induction-reaction model using the detailed reaction mechanism.

The basic of constructing a reaction model is that the key detonation combustion parameters are consistent with the results of the detailed reaction mechanism. For a given combustible mixture, the two-step reaction model parameters include: specific total energy $Q$, specific heat ratio $\gamma$, activation energy of the induction zone $E_{I}$, activation energy of the reaction zone $E_{R}$, pre-exponential factor of the reaction zone $k_{R}$, gas constant $R$, and physical length of the induction zone $\Delta_{\mathrm{I}}$. The detonation velocity, Mach number and the degree of stability are the key parameters in this study. Hence, the relationship between the two-step model and the detailed reaction mechanism can be obtained using one-dimensional detonation combustion theory with the constant specific heat ratio. The C-J detonation Mach number $M_{C J}$ is given by:

$M_{C J}=\left[\left(1+\frac{\gamma^{2}-1}{\gamma} Q\right)+\sqrt{\left[\left(1+\frac{\gamma^{2}-1}{\gamma} Q\right)^{2}-1\right]}\right]^{1 / 2}$

where $M_{C J}$ is defined as:

$$
\mathrm{M}_{\mathrm{CJ}}=\frac{\mathrm{V}_{\mathrm{CJ}}}{\sqrt{\gamma R \mathrm{~T}_{0}}}
$$

The specific heat ratio of the von-Neumann state $\gamma_{\mathrm{vn}}$ can be used as the specific heat ratio $\gamma$ of the two-step model, as demonstrated in the previous study [36]. Consequently, the specific total energy $Q$ and the gas constant $R$ of the two-step reaction model can be obtained from Eqs. (11) and (12) for the given detonation parameters $\left(M_{C J}, V_{C J}, \gamma\right)$, which are obtained from the CANTERA chemical software using the detailed reaction mechanism.

The activation energy of the induction zone $E_{\mathrm{I}}$ is calculated using the constant volume combustion (CVC) theory and the detailed solution process have been described in the previous study [33]. There are two key pre-exponential factors, $k_{\mathrm{I}}$ and $k_{R}$, which are used to complete the chemistry model. In this study, $k_{\mathrm{I}}=-u_{\mathrm{vn}}$, where $u_{\mathrm{vn}}$ is the post-shock particle velocity in the shock-fixed frame for the CJ detonation, whereby the induction length of the one-dimensional detonation is fixed to unity. The factor of the heat release step $k_{\mathrm{R}}$ is employed as a bifurcation parameter to vary the reaction length scale. For the present chemical model of ideal gases with a constant specific heat ratio, the heat release rate is expressed as:

$\dot{\sigma}=(\gamma-1) \frac{\mathrm{Q}}{\mathrm{c}^{2}} \frac{\mathrm{d} \lambda}{\mathrm{dt}}=\frac{\gamma-1}{\gamma} \mathrm{Q} \frac{(1-\lambda)}{\mathrm{T}} k_{\mathrm{R}} \exp \left(-\frac{E_{\mathrm{R}}}{\mathrm{T}}\right)$

Given the identical stability parameter $\chi$, the maximum rate is given by:

$\dot{\sigma}_{\max }=\frac{\gamma-1}{\gamma} \mathrm{Q} \frac{1}{\mathrm{~T}_{\mathrm{s}}} k_{\mathrm{R}} \exp \left(-E_{\mathrm{R}}\right)$

where $T_{S}$ is the post-shock temperature calculated based on the Rankine-Hugoniot relations.

$\mathrm{T}_{\mathrm{s}}=1+\frac{2(\gamma-1)\left(\gamma \mathrm{M}_{\mathrm{CJ}}^{2}+1\right)\left(\mathrm{M}_{\mathrm{CJ}}^{2}-1\right)}{\mathrm{M}_{\mathrm{CJ}}^{2}(\gamma+1)^{2}}$

For a given combustible mixture, the maximum heat release rate is easily computed using the detailed reaction mechanism. Hence, the relationship between the activation energy $E_{R}$ and the pre-exponential factor $k_{R}$ is obtained. Considering a flight altitude of $18 \mathrm{~km}(p=7565.2 \mathrm{~Pa}$, $\mathrm{T}=216.7 \mathrm{~K}$ ) and a stoichiometric hydrogen-air mixture, the main parameters are:

$Q=25.31, \gamma=1.32, E_{I}=6.52 T_{S}, E_{R}=1.00 T_{S}, k_{R}=3.74$

The main combustion parameters of the detailed reaction mechanism and two-step model are shown in Table 1.

\section{Basic structure and resolution study}

The RDW flow field contours at four different $\mathrm{T}_{\text {st }}$ are simulated and shown in Fig. 2. The grid scale is 0.2 and the grid number is $1200 \times 1800$. The common structure is observed to consist of an oblique detonation, an oblique shock induced by the detonation, and the contact surface between the fresh mixtures and products. An unstable slip line is generated in the detonation product due to the Kelvin-Helmholtz $(\mathrm{K}-\mathrm{H})$ instability. These structures agree well with the results of the previous study [6] and moreover, it is observed that $T_{\text {st }}$ has a significant effect on the formation of multiple detonation fronts. An increase in the $\mathrm{T}_{\text {st }}$ from 3.8 to 3.9 does not change

\begin{tabular}{|c|c|c|}
\hline Parameters & Detailed & Two-step \\
\hline $\mathrm{V}_{\mathrm{CJ}}(\mathrm{m} / \mathrm{s})$ & 1929.5 & 1929.5 \\
\hline$M_{\mathrm{CJ}}$ & 5.54 & 5.54 \\
\hline $\mathrm{T}_{\mathrm{CJ}}(\mathrm{K})$ & 2760.8 & 2266.7 \\
\hline$p_{\mathrm{CJ}}(\mathrm{kPa})$ & 155.5 & 135.6 \\
\hline $\mathrm{T}_{\mathrm{vn}}(\mathrm{K})$ & 1419.4 & 1263.4 \\
\hline$P_{\mathrm{vn}}(\mathrm{kPa})$ & 275.8 & 263.6 \\
\hline$\gamma_{0}$ & 1.41 & 1.32 \\
\hline$\gamma_{C J}$ & 1.25 & 1.32 \\
\hline$R_{0}(\mathrm{~J} /(\mathrm{kg} \cdot \mathrm{K}))$ & 397.6 & 422.8 \\
\hline$R_{\mathrm{CJ}}(\mathrm{J} /(\mathrm{kg} \cdot \mathrm{K}))$ & 351.2 & 422.8 \\
\hline
\end{tabular}




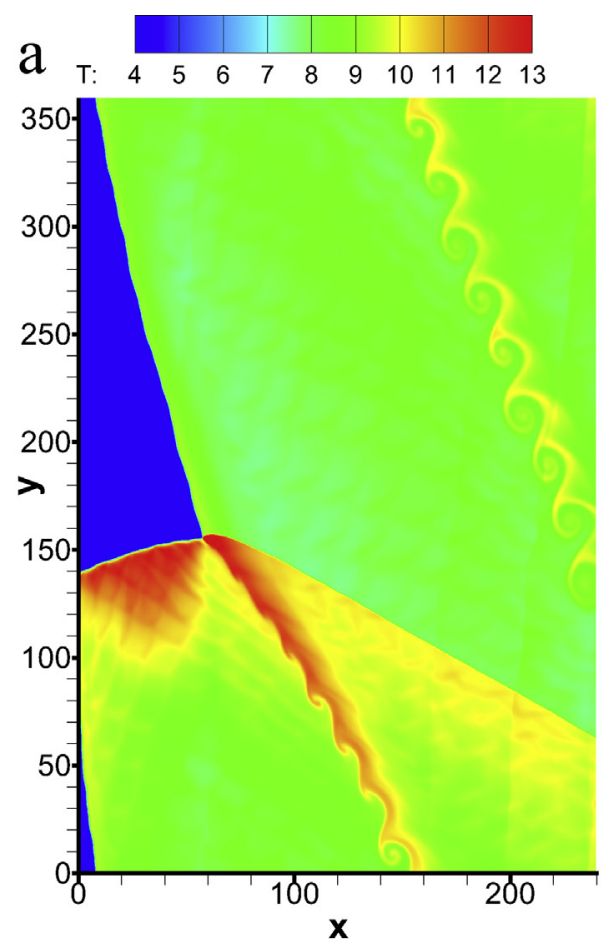

$\mathrm{b}$

C

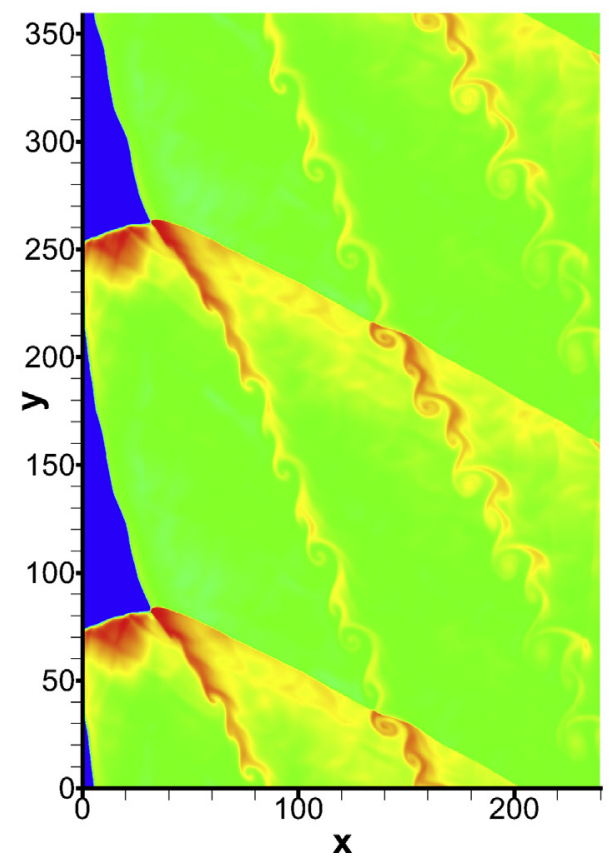

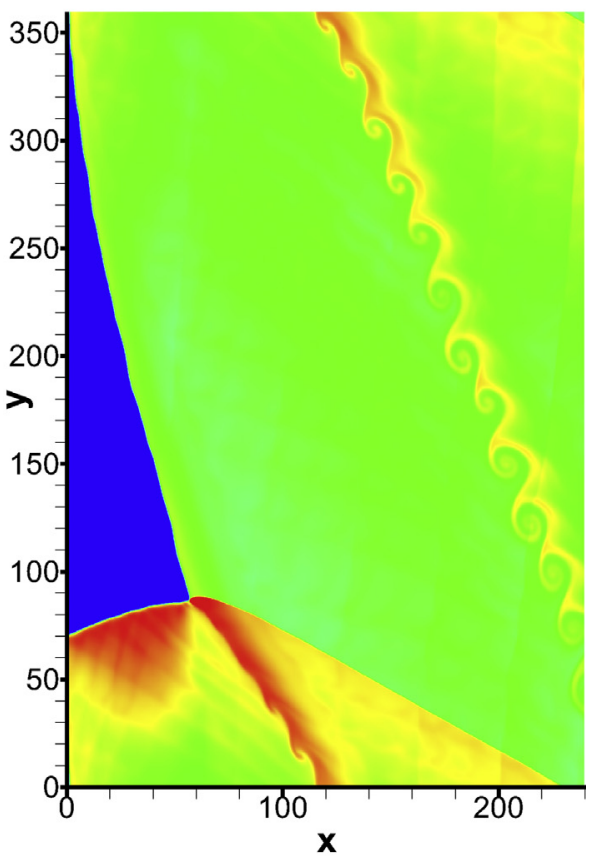

d

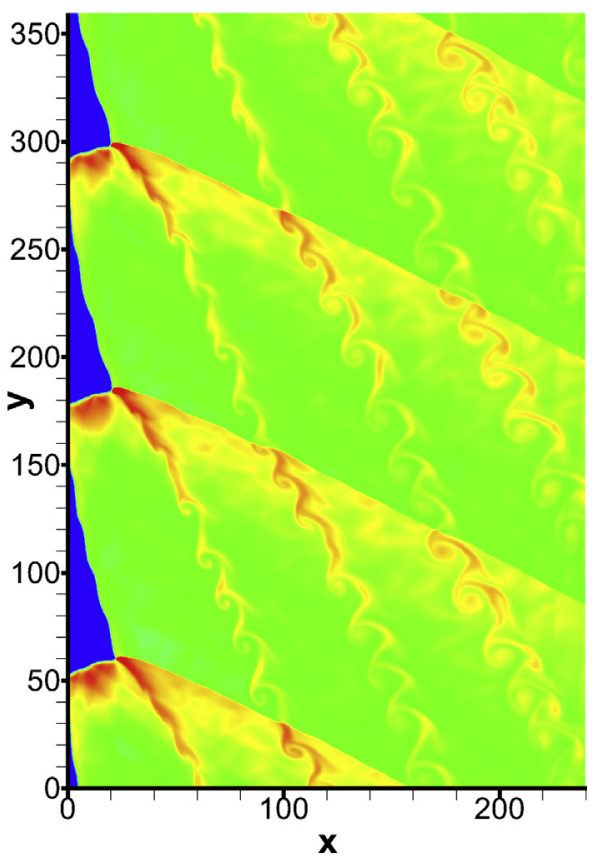

Fig. 2 - Temperature fields of RDW with $T_{s t}=3.8$ (a), 3.9 (b), 4.0 (c), and 4.1 (d).

the wavelet number. However, a further increase in the $T_{\text {st }}$ from 3.9 to 4.1 results in an increase in the number of detonations from one to three and a decrease in the height of wave front. It is noteworthy that all the results use the same initiation region and the multiple detonations are formed due to the interactions between the jet flow and the initial shock waves induced by the ignition zone. Generally, a pre- detonation tube or hot spot is used to initiate the RDWs; therefore, it is impossible to accurately simulate the initiation processes. Thus, this study focuses on the macroscopic structures of flow fields that converge to a quasi-steady state as time.

A grid-dependency study is conducted to compare the results produced by two grid scales, i.e. 0.2 and 0.1 . The 


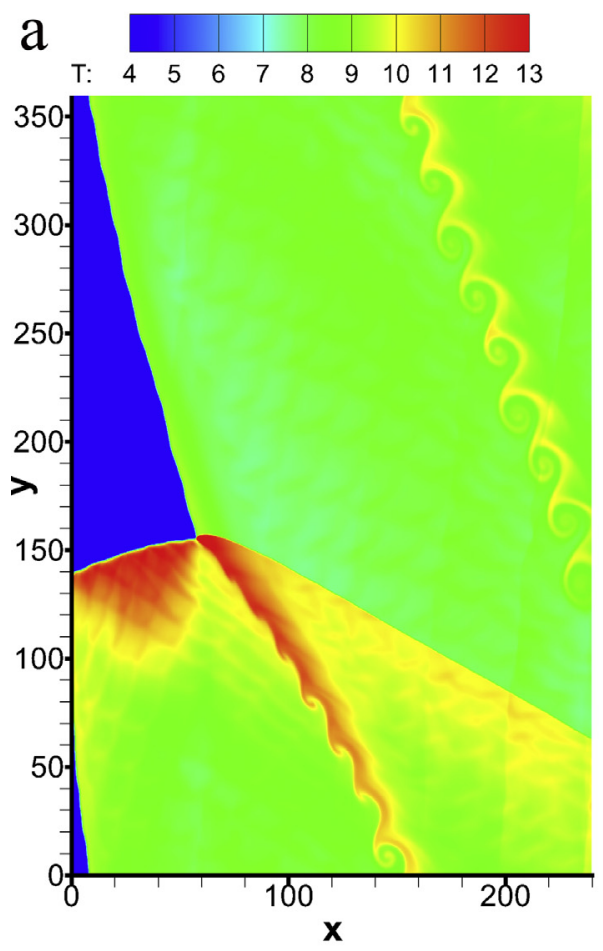

b

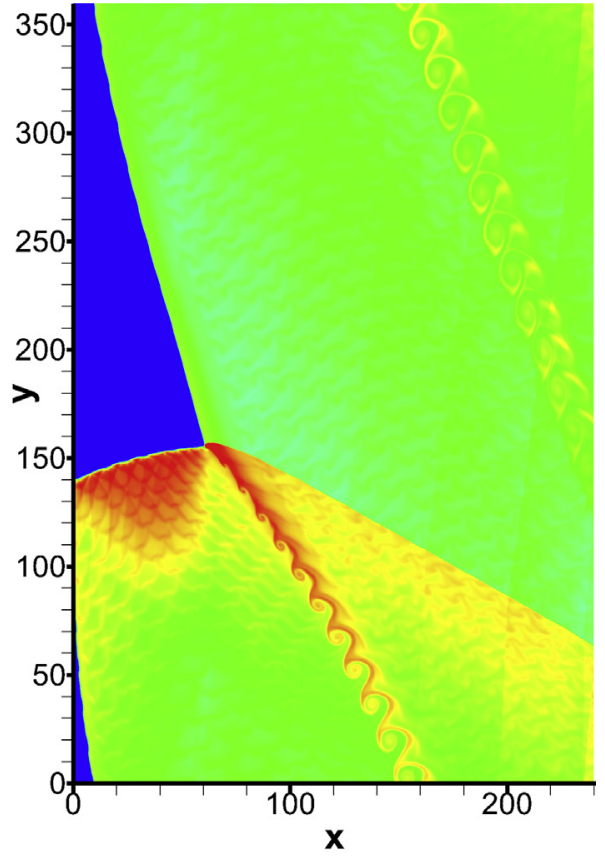

C
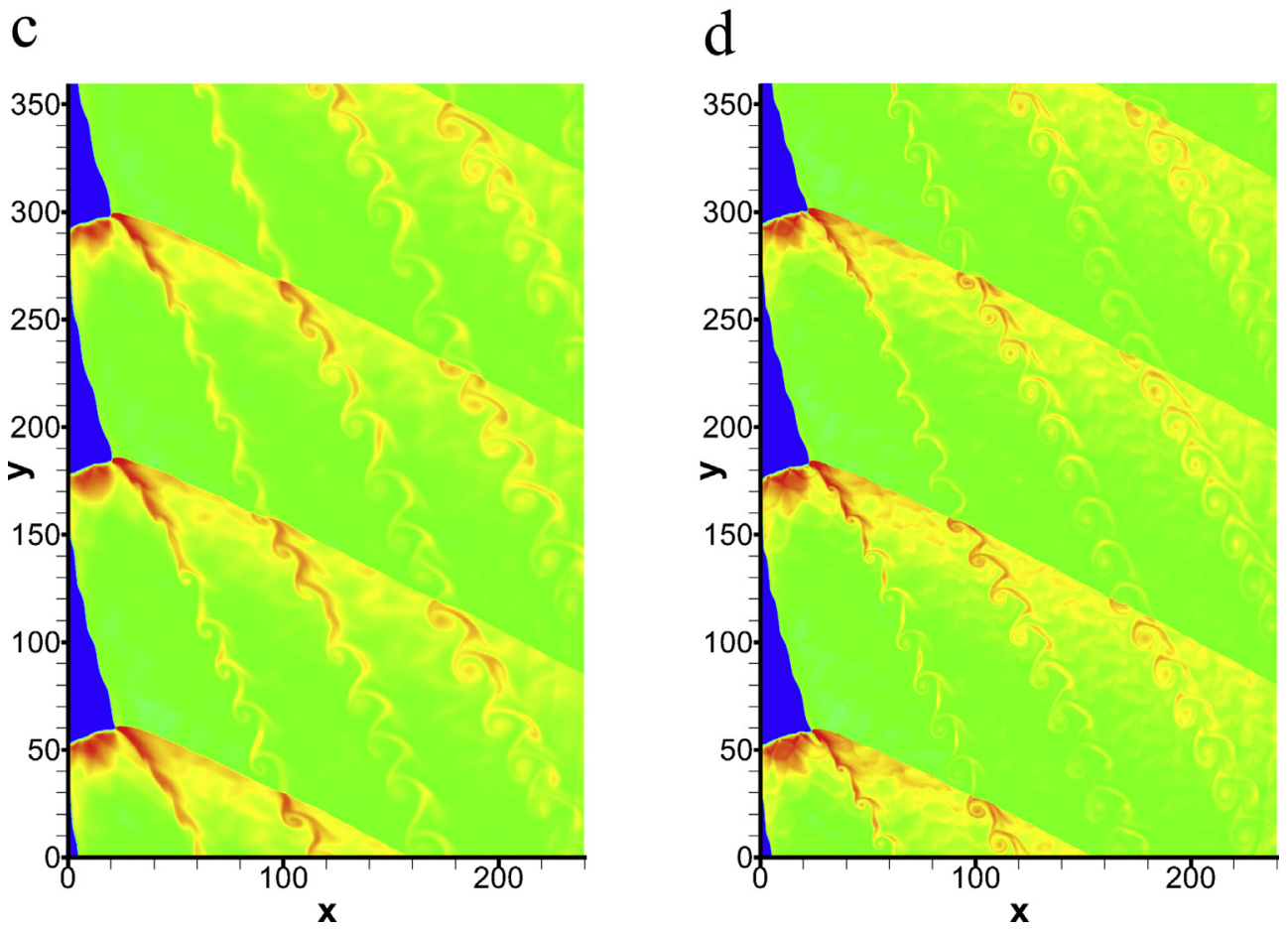

Fig. 3 - Temperature fields of the RDW, (a) $\mathrm{T}_{\mathrm{st}}=3.8$, grid $0.2 \times 0.2$; (b) $\mathrm{T}_{\mathrm{st}}=3.8$, grid $0.1 \times 0.1 ;$ (c) $\mathrm{T}_{\mathrm{st}}=4.1$, grid $0.2 \times 0.2$; (d) $\mathrm{T}_{\mathrm{st}}=4.1$, grid $0.1 \times 0.1$.

single and triple waves of RDW flow fields are both calculated and shown in Fig. 3. For gird comparison, the two grid scales are both sufficient to capture the features of the RDWs, even the contact surface's instability. Although the flow field contours provides more details on the $\mathrm{K}-\mathrm{H}$ instability using a finer grid scale, it has little effect on the thermodynamics parameters of the flow, such as temperature and pressure. The focus of this study is the macroscopic structure of the RDWs, such as the number of the detonations, not the microstructure. Hence, the grid scale $d x=0.20$ could provide reliable results and is used subsequently in this paper. 


\section{Effects of heat release rate}

Although the chemical parameters described in Modeling the chemical reactions using two-step kinetics correspond to a specific flight conditions, it is rewarding to perform the effects of different fuels on the RDW structure in a qualitative manner by varying $k_{\mathrm{R}}$. The consequence of varying $k_{\mathrm{R}}$ is a change of the induction-to-reaction length ratio, which is known to affect the gaseous detonation surface's stability
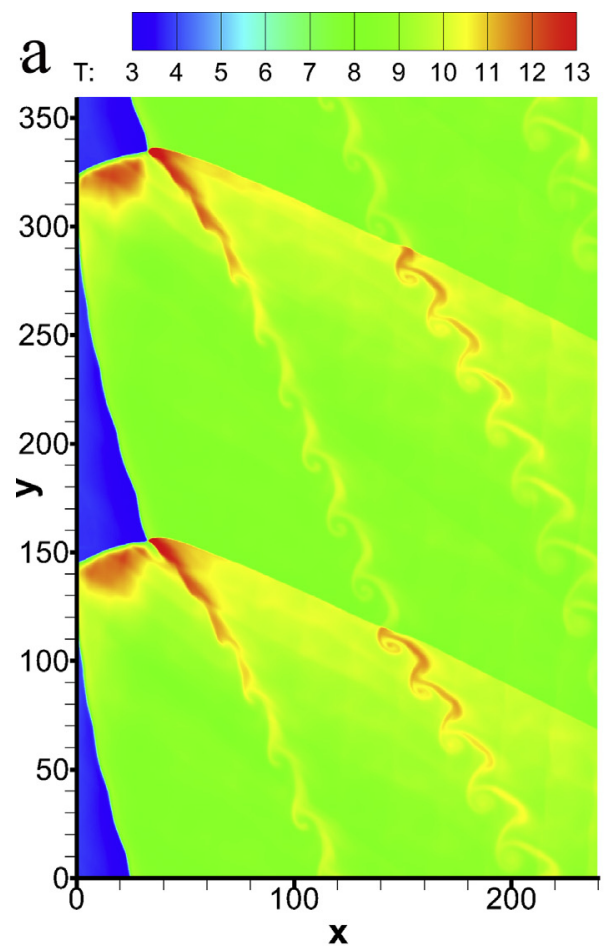

C

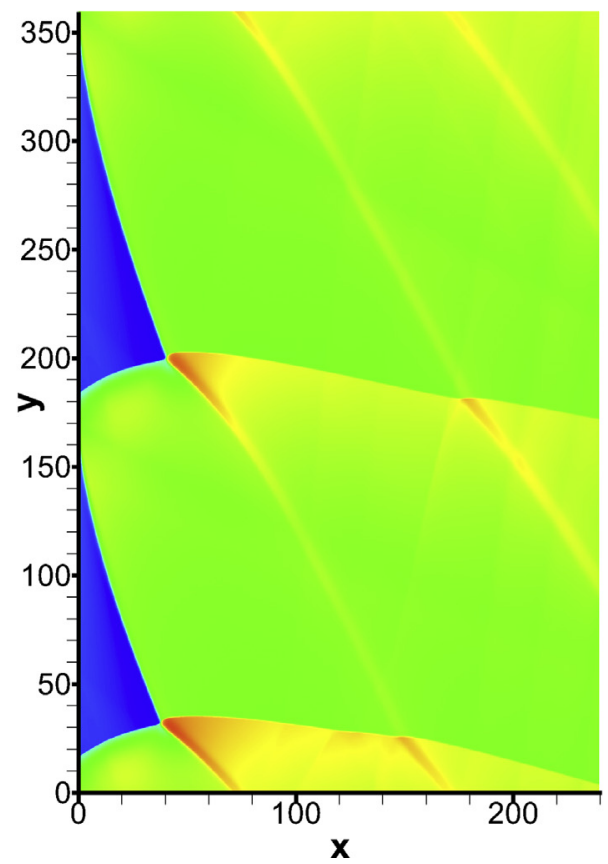

[32,33]. The rate $k_{R}$ controls the degree of coupling between the leading shock and heat release front, and plays a vital role in detonation initiation and propagation.

Fig. 4 shows the temperature field of the RDW at the temperature $T_{\text {st }}=4.1$ with varying $k_{R}$ (from 2.0 to 0.4 ). Unlike the results shown in Fig. $2 d\left(k_{R}=3.74\right)$, the number of detonation waves decreases when $k_{R}$ changes from 3.74 to 2.0, which results in a slight increase in the wavelet height. For a further decrease in $k_{\mathrm{R}}$ from 2.0 to 0.4 , there is no

b

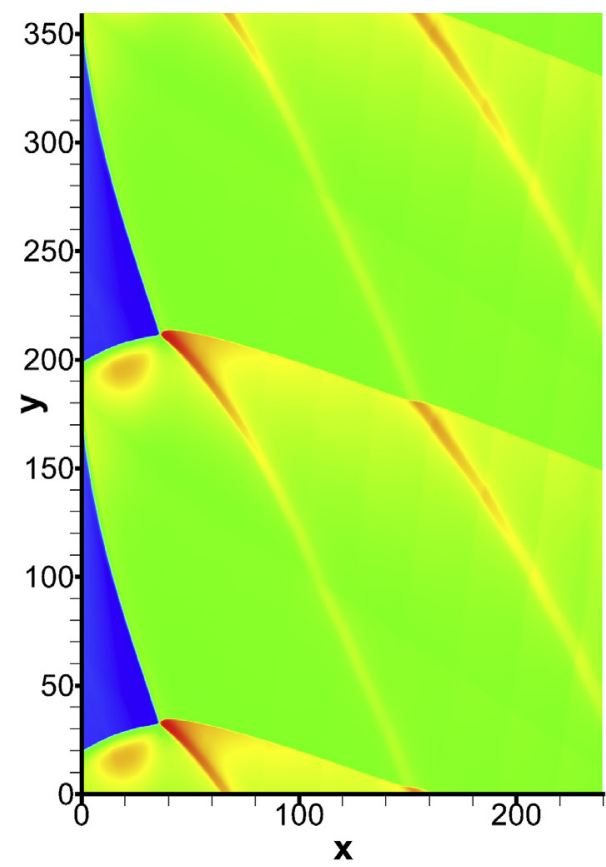

d

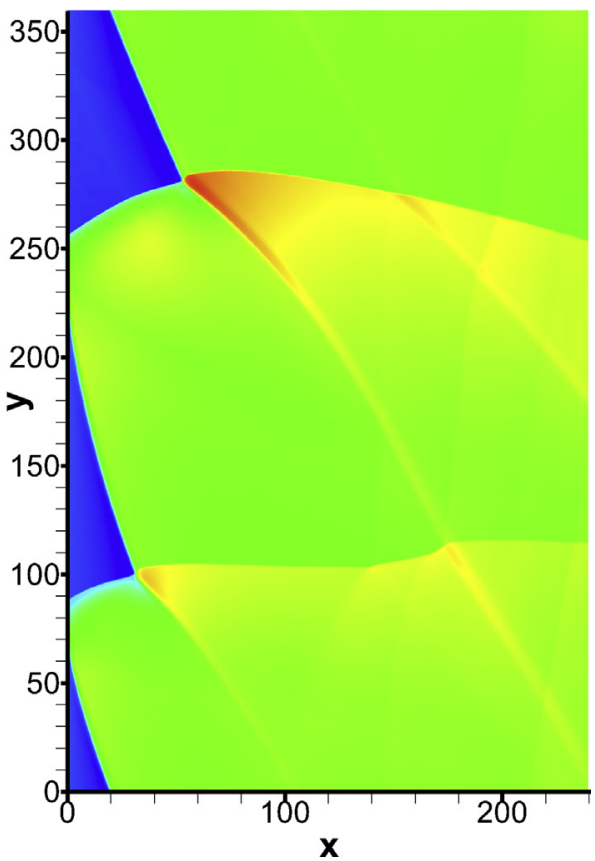

Fig. 4 - Temperature field of the RDW with $T_{s t}=4.1$ and $k_{R}=2.0$ (a), 1.0 (b), 0.5 (c), and 0.4 (d). 
change in the wavelet number, but the surface of the detonation wave becomes smoother and the $\mathrm{K}-\mathrm{H}$ instability of the contact surface is observed to fade away. Previous studies [31-33] also found that the detonation front becomes stable when $k_{\mathrm{R}}$ decreases using the two-step induction-reaction model. However, it is interesting that the differences in the two wavelets increase as $k_{\mathrm{R}}$ decreases from 1.0 to 0.4 , as shown in Fig. $4 \mathrm{~b}$ and $\mathrm{d}$. The detonation pressure and height experience high-amplitude oscillations through the operating cycle. These results present the presence of another instability mechanism that may influences the wavelet behavior, and this phenomenon deserves more attention.
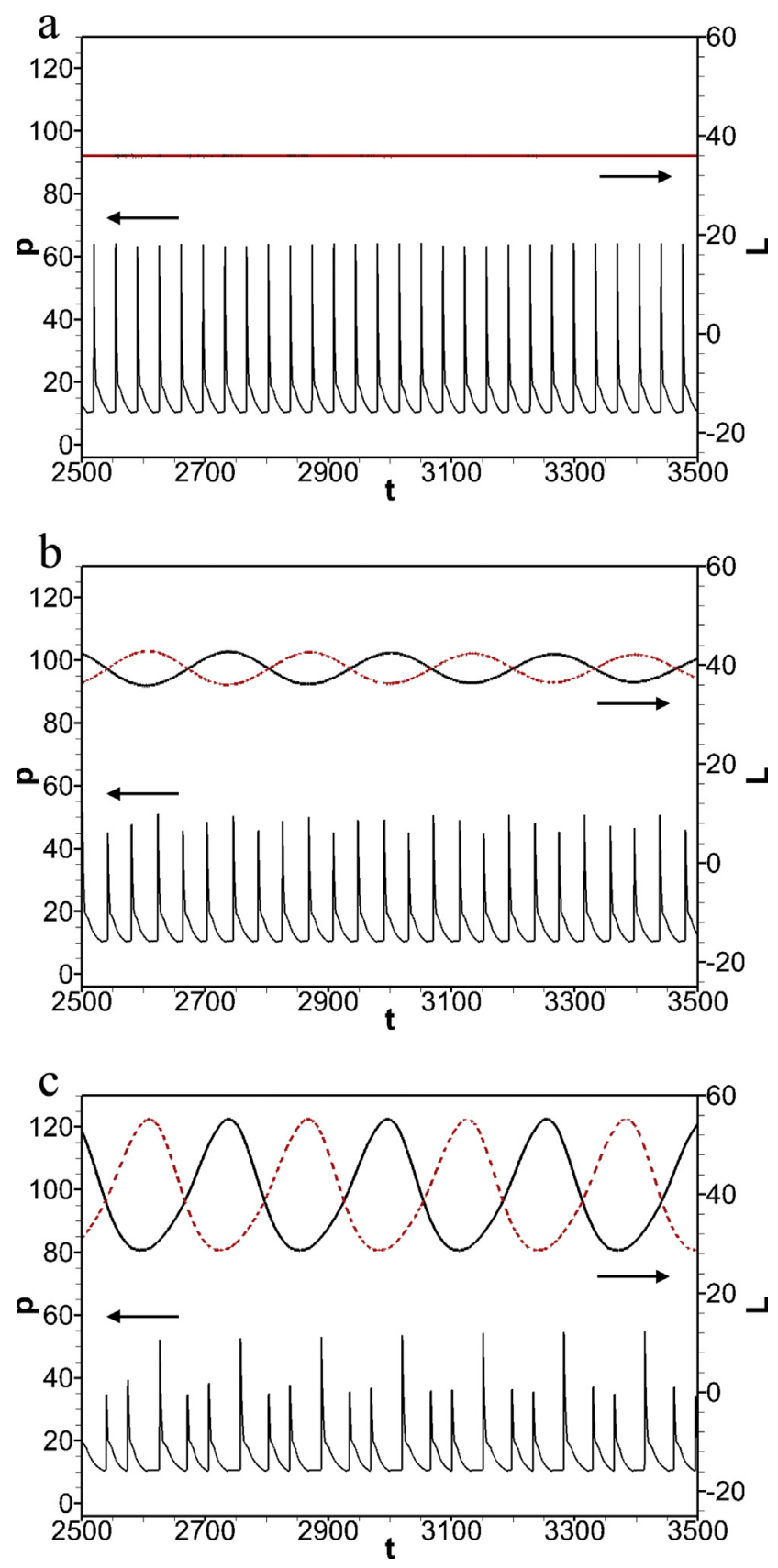

Fig. 5 - Evolution of detonation wavelet height and the pressure with $T_{s t}=4.1$ and $k_{R}=1.0(a), 0.5(b)$, and 0.4 (c).
To gain an understanding of the evolution of multiple detonations and the underlying mechanism, the long-time simulations are performed to eliminate the effects of initiation. Fig. 5 shows the wavelet height and the pressure at a preset point for three reaction rate $\left(k_{\mathrm{R}}=1.0,0.5,0.4\right)$. The data are recorded after the successful initiation and a long-time iteration is used to reach a quasi-steady state. According to the computational results in this paper, the height of the multiple waves in a combustor can maintain the same value for a high reaction rate $\left(k_{R} \geq 1.0\right)$. The detonation transitions from a quasi-steady state to an undulate operation as the reaction rate is further decreased (to 0.5 ) resulting in an oscillation in the corresponding detonation height/pressure. It is observed that the amplitude of height oscillation decays slowly, therefore, the heights of the two wavelets approach the same value as time. However, when $k_{\mathrm{R}}$ decreases to 0.4 , the height of the detonation wavelets ceases to decay anymore and the oscillation of the detonation height/pressure with strong regularity is observed.

As mentioned above, the decrease in the rate $k_{\mathrm{R}}$ suppresses the instability of the detonation front, which is usually reflected in the disappearance of transverse waves originating from triple shock interactions. However, the decreased $k_{\mathrm{R}}$ results in a decrease in stability of the multiple wave configurations, which presents the oscillation of the detonation height/pressure. The stability parameter $\chi$, which is defined by the ratio of the induction length and the reaction length multiplied by the global reduced activation energy, does not explain this physical phenomenon accurately and thoroughly. This is because that the parameter $\chi$ only depicts the instability of the detonation surfaces, whereas the unstable RDW flow fields include the interaction of detonations, shock waves and heat release. There exist plenty of expansion waves behind the oblique shock which is induced by the detonation to the pressure of the products. The expansion waves weaken the detonation front, especially for a low values of $k_{\mathrm{R}}$. A decrease in $k_{\mathrm{R}}$ results in a long width of the heat release zone, which leads to the reduction of chemical energy used to sustain the propagation of the leading shock. The detonation capacity of resisting disturbance decreases, and hence the expansion waves can weaken significantly the detonation and cause the oscillations of the detonation height and pressure for the low values of the rate $k_{R}$.

\section{Possibility of multi-pattern induced by initiation}

The RDW flow fields are composed of complex wave configurations and varied chemical reaction processes. The patterns of the RDWs after ignition may be affected by the initial conditions. To intuitively present the possibility of multi-pattern waves in a rotating detonation combustor, the initial flow is ignited using three ignition methods (see Fig. 1, i.e., $L_{1} \times L_{2}=48 \times 150 ; L_{1} \times L_{2}=192 \times 150 ; L_{1} \times L_{2}=192 \times 250$, named as weak ignition, medium ignition and strong ignition respectively) in this section. Fig. 6 shows the temperature fields of the steady RDWs with the three different ignition zones at the same injection stagnation temperature $T_{\text {st }}=4.1$. These results reveal an interesting phenomenon occurring during the formation of the multiple detonations. For the medium ignition case, there exist three detonation waves in a 


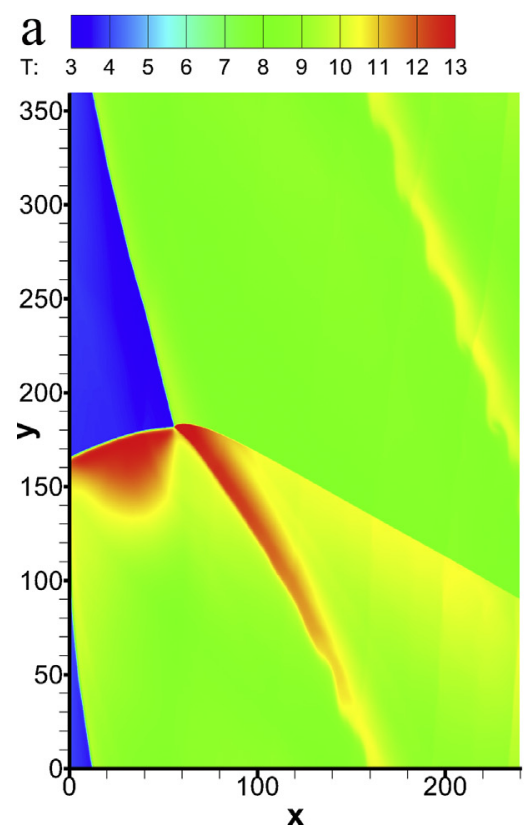

$\mathrm{b}$

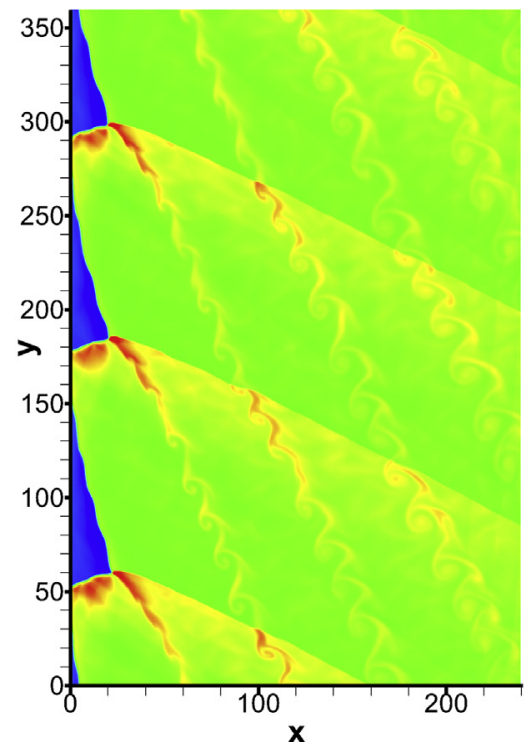

$\mathrm{C}$

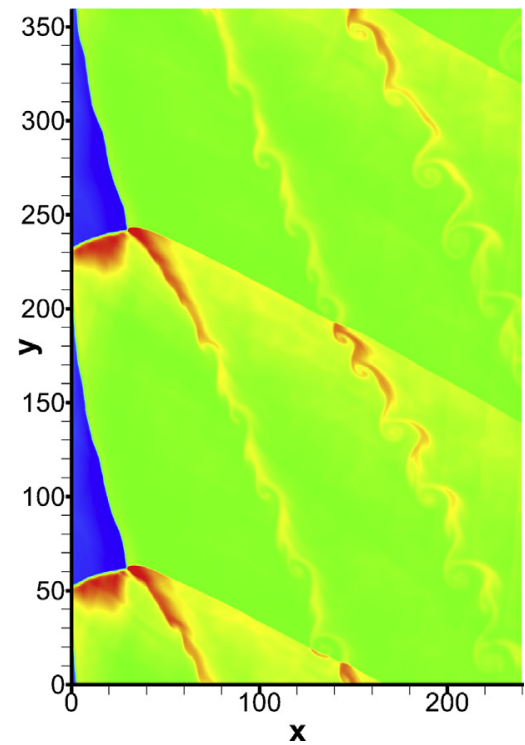

Fig. 6 - Temperature field with $T_{s t}=4.1$ and different initiation patterns: weak ignition (a), medium ignition (b) and strong ignition (c).

combustor, whereas the wave numbers have a decrease for the two other ignitions. This indicates that the dependence of multiple waves on the ignition patterns is non-linear.

Compared to the results after the weak ignition (Fig. 6a), the ignition zone in Fig. $6 \mathrm{~b}$ has a wider scale in the $x$-direction and the initial free flow has the same thermodynamic parameters. Generally, the greater initiation energy will be propitious to the formation of the multiple detonations and could supports more waves in the initial stage for a confined space, which are agree well with the results shown in Fig. 6a and b. However, it is worth noting that even though the strong ignition has greater ignition area, there are only two waves in the entire flow (Fig. $6 \mathrm{c}$ ). These results may be a little different from the viewpoint mentioned above, while this paradox can be explained as follows. The ignition zone is an ideal C-J detonation wave which is obtained in a one dimensional channel. The channel is open at both ends and hence the tail of the ignition zone contains a large number of rarefaction waves. Compared to the medium ignition, the strong ignition has greater ignition area and more rarefaction waves at the end of $C J$ detonation. The rarefaction waves reduce the concentrated release of energy and repress the formation of multiple detonation waves. Consequently, there exist two detonation waves in the combustor after the strong ignition. Under some conditions, many previous experimental studies $[11,12,37]$ have showed there exist various operating modes in the combustor even with the same geometric conditions and injection parameters. Even in one experiment, there could be a sudden change of propagation mode (defined by the number of detonation waves), which suggests the presence of a strong instability associated with the detonation combustion. These results indicate that the co-existence of different wavelet configurations in a combustor. For the simulation cases, the ignition patterns could trigger the strong instability of RDWs, which results in the various operation modes. Although the comparisons between the present simulation results and the experimental features are intuitive, these results conform to the laws of physics and can be construed as a physical homeostasis.

In addition, the generalizing dependences of the RDW flow structures on the stagnation temperature and the initiation pattern are shown in Fig. 7. The $x$-axis and $y$-axis represent the stagnation temperature $T_{\text {st }}$ and the number of detonation waves, respectively. In general, these results indicate that the higher stagnation temperature $T_{\text {st }}$ promotes the formation of multiple waves and result in various forms of the RDW flow field. Furthermore, the RDW flow structures are sensitive to

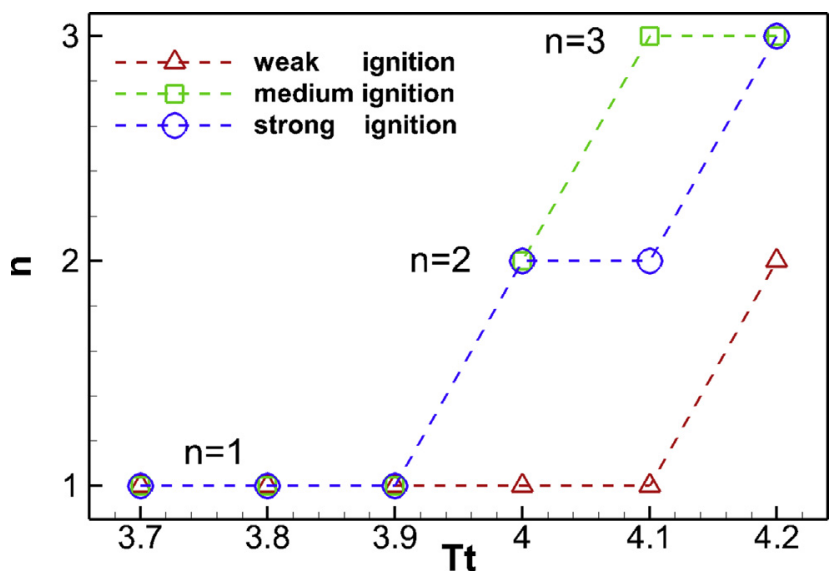

Fig. 7 - Generalized dependences of the number of detonation waves on the stagnation temperature and the initiation pattern. 
the ignition patterns and there are various quasi-steady patterns at high stagnation temperatures.

Previous studies $[2,3,28,29]$ have demonstrated that the number of the detonation increases as the air mass flow rate is increased. The variation of $\mathrm{T}_{\text {st }}$ has little difference on the mass flow rate for the given computational parameters in this study. The wavelet number and height evolution cannot be explained from the point of mass flow rate, and hence the coupling of flow dynamics and heat release plays a noticeable role in wave stability and initiation partly. Researchers [37] have shown that an increase in the number of detonation waves in the combustor depends on the normalized perimeter $(p / \lambda)$ of the detonation wave. As the value $(p / \lambda)$ increases to a critical value, the number of detonation waves triggers a transition to a more stable operation. For the stable RDW in Fig. $2 a$ and $b$, the detonation propagates stably and the height and intensity of the detonation wave tends to be stable as time. When the stagnation temperature $T_{\text {st }}$ is further increased to high value, the RDW transitions from one-wave to two/three-wave operation. The reaction rate is sensitive to the reactants temperature. The chemical reactivity of the mixtures is weak at low temperature, which makes the corresponding RDW flow structures more stable. Hence, the steady propagation mode is independent of the ignition patterns below the critical total temperature. The increased temperature improves the inherent instability of the entire RDW flow and breaks the balance of the wave configurations, which is more sensitive to the initial conditions to a great extent. For the strong unstable cases with a high stagnation temperature, a decrease in the heat release rate $k_{R}$ promotes the decoupling of the shock and reaction front and exacerbates the instability of the entire flow. Consequently, the pressure of the pre-set point and the height of the wavelet oscillate periodically.

\section{Conclusion}

Two-dimensional RDWs are simulated to investigate the effects of the inflow stagnation temperature, the initiation pattern and the heat release rate on the wavelet features. The results indicate the generalizing dependences of the number of RDWs on the thermodynamic parameters and the instability of multiple waves using a two-step induction-reaction model.

The basic results are agree well with previous studies that the RDWs consist of a series of wave structures, such as detonations, shocks, slip line and the contact surface, etc. By increasing the stagnation temperature $T_{s t}$, it is found that there exists various operating modes in a combustor, like single-wave, double-waves and triple-wave operation. An interesting phenomenon is observed in the number, height and pressure of the detonation as the heat release rate $k_{R}$ is further decreased. These results indicate the effect of the coupling between the flow and heat release on the instability of the RDW flow field. A further decrease in the rate $k_{R}$ also results in the disappearance of the triple points of the rotating detonation surface, which are agree well with previous studies [32,33]. However, the entire flow structures become more unstable and the detonation pressure/height changes periodically at a low value of $k_{R}$. The multi-wave structure of the rotating detonation may be affected by many factors. The exact mechanism behind the formation and mode switching of RDWs in the combustor is still unclear. But we demonstrate the possibility of multiple patterns induced by initiations and identify the co-existence of different wave configurations in a combustor, in which three ignition patterns are used to obtain the various propagation modes. Concurrently, the results also show that wavelet features of the rotating detonation could be more sensitive to the ignition pattern at high stagnation temperature $T_{\text {st }}$.

Lastly, the propagation of multiple RDWs in a combustion chamber induces complex phenomena, including the formation of multiple waves, the interference of the shock wave and detonation, and the coupling of the flow and heat release, etc. A basic understanding of the rotating detonation is not only helpful to understand the physical mechanism but also for engineering applications, and hence more fundamental works on RDWs are required in future.

\section{Acknowledgement}

The research is supported by the National Natural Science Foundation of China NSFC Nos. 11822202, 91641130.

\section{R E F E R E N C E S}

[1] Wolański P. Detonative propulsion. Proc Combust Inst 2013;34:125-58. https://doi.org/10.1016/j.proci.2012.10.005.

[2] Bykovskii FA, Zhdan SA, Vedernikov EF. Continuous spin detonations. J Propuls Power 2006;22:1204-16. https:// doi.org/10.2514/1.17656.

[3] Bykovskii FA, Zhdan SA. Current status of research of continuous detonation in fuel-air mixtures (Review). Combust Explos Shock Waves 2015;51:21-35. https://doi.org/ 10.1134/S0010508215010025.

[4] Lu FK, Braun EM. Rotating detonation wave propulsion: experimental challenges, modeling, and engine concepts. J Propuls Power 2014;30:1125-42. https://doi.org/10.2514/ 1.B34802.

[5] Rankin BA, Fotia ML, Naples AG, Stevens CA, Hoke JL, Kaemming TA, Theuerkauf SW, Schauer FR. Overview of performance, application, and analysis of rotating detonation engine technologies. J Propuls Power 2017;33:131-43. https://doi.org/10.2514/1.B36303.

[6] Kailasanath $\mathrm{K}$. Recent developments in the research on rotating-detonation-wave engines. In: 55th AIAA aerospace sciences meeting. Texas: Grapevine; 2017. https://doi.org/ 10.2514/6.2017-0784. AIAA 2017-0784.

[7] Anand V, Gutmark E. Rotating detonation combustors and their similarities to rocket instabilities. Prog Energy Combust Sci 2019;73:182-234. https://doi.org/10.1016/j.pecs.2019.04.001.

[8] Schwer DA, Kailasanath K. Numerical study of the effects of engine size on rotating detonation engines. In: 49th AIAA aerospace sciences meeting including the new horizons forum and aerospace exposition; 2011. https://doi.org/ 10.2514/6.2011-581. AIAA 2011-581, Orlando, Florida.

[9] Zhou R, Wang J. Numerical investigation of shock wave reflections near the head ends of rotating detonation engines. Shock Waves 2013;23:461-72. https://doi.org/ 10.1007/s00193-013-0440-0. 
[10] Katta VR, Cho KY, Hoke JL, Codoni JR, Schauer FR, Roquemore WM. Effect of increasing channel width on the structure of rotating detonation wave. Proc Combust Inst 2019;37:3575-83. https://doi.org/10.1016/j.proci.2018.05.072.

[11] Lin W, Zhou J, Liu S, Lin Z, Zhuang F. Experimental study on propagation mode of $\mathrm{H} 2 /$ Air continuously rotating detonation wave. Int J Hydrogen Energy 2015;40:1980-93. https://doi.org/10.1016/j.ijhydene.2014.11.119.

[12] Anand V, St George A, Driscoll R, Gutmark E. Characterization of instabilities in a rotating detonation combustor. Int J Hydrogen Energy 2015;40:16649-59. https:// doi.org/10.1016/j.ijhydene.2015.09.046.

[13] Yang C, Wu X, Ma H, Peng L, Gao J. Experimental research on initiation characteristics of a rotating detonation engine. Exp Therm Fluid Sci 2016;71:154-63. https://doi.org/10.1016/ j.expthermflusci.2015.10.019.

[14] Zhou S, Ma H, Liu D, Yan Y, Li S, Zhou C. Experimental study of a hydrogen-air rotating detonation combustor. Int J Hydrogen Energy 2017;42:14741-9. https://doi.org/10.1016/ j.ijhydene.2017.04.214.

[15] Zhou S, Ma H, Liu C, Zhou C, Liu D. Experimental study of a hydrogen-air rotating detonation engine with variable airinlet slot. Int J Hydrogen Energy 2018;43:11253-62. https:// doi.org/10.1016/j.ijhydene.2018.05.033.

[16] Experimental investigation on propagation characteristics of rotating detonation wave with a hydrogen-ethyleneacetylene fuel. Acta Astronaut 2019;157:310-20. https:// doi.org/10.1016/j.actaastro.2019.01.009.

[17] Xia Z, Ma H, Zhuo C, Zhou C. Propagation process of H2/air rotating detonation wave and influence factors in planeradial structure. Int J Hydrogen Energy 2018;43:4609-22. https://doi.org/10.1016/j.ijhydene.2018.01.061.

[18] Xia ZJ, Tang XM, Luan MY, Zhang SJ, Ma Z, Wang JP. Numerical investigation of two-wave collision and wave structure evolution of rotating detonation engine with hollow combustor. Int J Hydrogen Energy 2018;43:21582-91. https://doi.org/10.1016/j.ijhydene.2018.09.165.

[19] Bluemner R, Bohon MD, Paschereit CO, Gutmark EJ. Counterrotating wave mode transition dynamics in an RDC. Int $J$ Hydrogen Energy 2019;44:7628-41. https://doi.org/10.1016/ j.ijhydene.2019.01.262.

[20] Sosa J, Ahmed KA, Fievisohn R, Hoke J, Ombrello T, Schauer F. Supersonic driven detonation dynamics for rotating detonation engines. Int J Hydrogen Energy 2019;44:7596-606. https://doi.org/10.1016/ j.ijhydene.2019.02.019.

[21] Huang S, Li Y, Zhou J, Liu S, Peng H. Effects of the pintle injector on $\mathrm{H} 2 /$ air continuous rotating detonation wave in a hollow chamber. Int J Hydrogen Energy 2019;44:14044-54. https://doi.org/10.1016/j.ijhydene.2019.04.011.

[22] Yamada T, Hayashi K, Tsuboi N, Yamada E, Tangirala V, Fujiwara T. Numerical analysis of threshold of limit detonation in rotating detonation engine. In: 48th AIAA aerospace sciences meeting including the new horizons forum and aerospace exposition; 2010. https://doi.org/ 10.2514/6.2010-153. AIAA 2010-153 Orlando, Florida.

[23] Tsuboi N, Watanabe Y, Kojima T, Hayashi AK. Numerical estimation of the thrust performance on a rotating detonation engine for a hydrogen-oxygen mixture. Proc
Combust Inst 2015;35:2005-13. https://doi.org/10.1016/ j.proci.2014.09.010.

[24] Schwer D, Kailasanath K. Numerical investigation of the physics of rotating-detonation-engines. Proc Combust Inst 2011;33:2195-202. https://doi.org/10.1016/j.proci.2010.07.050.

[25] Schwer D, Kailasanath K. Fluid dynamics of rotating detonation engines with hydrogen and hydrocarbon fuels. Proc Combust Inst 2013;34:1991-8. https://doi.org/10.1016/ j.proci.2012.05.046.

[26] Uemura Y, Hayashi AK, Asahara M, Tsuboi N, Yamada E. Transverse wave generation mechanism in rotating detonation. Proc Combust Inst 2013;34:1981-9. https:// doi.org/10.1016/j.proci.2012.06.184.

[27] Liu Y, Zhou W, Yang Y, Liu Z, Wang J. Numerical study on the instabilities in $\mathrm{H}_{2}$-air rotating detonation engines. Phys Fluids 2018;30:046106. https://doi.org/10.1063/1.5024867.

[28] Rankin BA, Richardson DR, Caswell AW, Naples AG, Hoke JL, Schauer FR. Chemiluminescence imaging of an optically accessible non-premixed rotating detonation engine. Combust Flame 2017;176:12-22. https://doi.org/10.1016/ j.combustflame.2016.09.020.

[29] Rankin BA, Codoni JR, Cho KY, Hoke JL, Schauer FR. Investigation of the structure of detonation waves in a nonpremixed hydrogen-air rotating detonation engine using mid-infrared imaging. Proc Combust Inst 2019;37:3479-86. https://doi.org/10.1016/j.proci.2018.09.036.

[30] Teng H, Ng HD, Li K, Luo C, Jiang Z. Evolution of cellular structures on oblique detonation surfaces. Combust Flame 2015;162:470-7. https://doi.org/10.1016/ j.combustflame.2014.07.021.

[31] Yang P, Teng H, Jiang Z, Ng HD. Effects of inflow Mach number on oblique detonation initiation with a two-step induction-reaction kinetic model. Combust Flame 2018;193:246-56. https://doi.org/10.1016/ j.combustflame.2018.03.026.

[32] Yang P, Teng H, Ng HD, Jiang Z. A numerical study on the instability of oblique detonation waves with a two-step induction-reaction kinetic model. Proc Combust Inst 2019;37:3537-44. https://doi.org/10.1016/j.proci.2018.05.090.

[33] Ng HD, Radulescu MI, Higgins AJ, Nikiforakis N, Lee JHS. Numerical investigation of the instability for onedimensional Chapman-Jouguet detonations with chainbranching kinetics. Combust Theor Model 2005;9:385-401. https://doi.org/10.1080/13647830500307758.

[34] Jiang ZL. On dispersion-controlled principles for nonoscillatory shock-capturing schemes. Acta Mech Sin 2004;20:1-15. https://doi.org/10.1007/BF02493566.

[35] Gamezo VN, Desbordes D, Oran ES. Formation and evolution of two-dimensional cellular detonations. Combust Flame 1999;116:154-65. https://doi.org/10.1016/S0010-2180(98) 00031-5.

[36] Radulescu MI, Maxwell BM. The mechanism of detonation attenuation by a porous medium and its subsequent reinitiation. J Fluid Mech 2011;667:96-134. https://doi.org/ 10.1017/S0022112010004386.

[37] George A St, Driscoll R, Anand V, Gutmark E. On the existence and multiplicity of rotating detonations. Proc Combust Inst 2017;36:2691-8. https://doi.org/10.1016/ j.proci.2016.06.132. 